\title{
A fixed strike Asian option and comments on its numerical solution
}

\author{
Jens Hugger*
}

(Received 8 August 2003, revised 31 March 2004)

\begin{abstract}
A boundary value formulation of an Asian option is solved with a wide range of standard textbook explicit finite difference methods including also artificial diffusion methods. We investigate the dependence of the numerical methods on the various degeneracies and approximations in the boundary value formulation. It is concluded that numerical solutions are generally oscillatory, that a simple artificial diffusion approach does not resolve this problem, but that by selecting the appropriate methods, oscillations can be avoided.
\end{abstract}

* Institute for Mathematical Sciences, University of Copenhagen, DK-2100 CPH, Denmark. mailto:hugger@math.ku.dk

See http://anziamj.austms.org.au/V45/CTAC2003/Hugg for this article, (C) Austral. Mathematical Soc. 2004. Published April 23, 2004. ISSN 1446-8735 


\section{Contents}

1 The boundary value problem for Asian options

C216

2 Simple textbook finite difference methods

C219

3 Numerical experiments and conclusions

$\mathrm{C} 224$

References

C230

\section{The boundary value problem for Asian options}

Most financial instruments (like contingent claims) are modelled within the framework of stochastics but have equivalent analytical formulations in the form of boundary value problems (BVP's) for partial differential equations.

For European options with many factors (more than 3), stochastic methods like Monte Carlo simulation may be the most competitive but for the more advanced options traded today and the more advanced numerical methods available the BVP formulations merits interest. For example this is the case for the Asian options considered in this paper.

We consider the Black-Scholes model of the reversed time formulation of a zero dividend, fixed strike Asian average value call option, where the terminal payoff depends on the path, that is, the past history of the risky asset, through the continuous arithmetic running sum (integral of the risky 
asset price over the entire lifetime of the option):

$$
\begin{aligned}
& \text { Find } V:(S, A, \tau) \in \bar{\Omega} \rightarrow \mathcal{R}, \quad V \in \mathcal{C}^{0}(\bar{\Omega}) \cap \mathcal{C}^{\infty}\left(\Omega_{\infty}\right) \text { with } \\
& \Omega=\left(0, S_{\max }\right) \times\left(0, A_{\max }\right) \times(0, T) \\
& \Omega_{\infty}=(0, \infty) \times(0, \infty) \times(0, T) \\
& \frac{\partial V}{\partial \tau}=\frac{\sigma^{2}}{2} S^{2} \frac{\partial^{2} V}{\partial S^{2}}+r S \frac{\partial V}{\partial S}+S \frac{\partial V}{\partial A}-r V \quad \text { for }(S, A, \tau) \in \Omega \\
& V(S, A, 0)=\max \left\{\frac{A}{T}-K, 0\right\} \\
& V(0, A, \tau)=e^{-r \tau} \max \left\{\frac{A}{T}-K, 0\right\}, \\
& V(S, A, \tau)=\left(\frac{A}{T}-K\right) e^{-r \tau}+\frac{S}{r T}\left(1-e^{-r \tau}\right), \quad \text { for } A \geq K T, \\
& V\left(S_{\max }, A, \tau\right) \simeq \max \left\{\left(\frac{A}{T}-K\right) e^{-r \tau}+\frac{S_{\max }}{r T}\left(1-e^{-r \tau}\right), 0\right\}
\end{aligned}
$$

See [1] and [2] for the derivation. The last boundary condition is exact only in the limit $S_{\max } \rightarrow \infty$. For notation, $t$ is time while $\tau=T-t$ is reversed time. The option is priced at time $t=0$ or $\tau=T$ and expires at the terminal time $t=T$ or $\tau=0$. The constants $r, \sigma>0$ and $K>0$ are the risk free market interest rate, volatility and the terminal exercise price respectively. $S_{\max }$ and $A_{\max }$ in the bounds of $\Omega$ constitute artificial limitations on the computational domain to facilitate numerical solution of the problem originally posed on the infinite domain $\Omega_{\infty} . V, S$ and $A$ are option price, risky asset price and risky asset price running sum $\left(A(t)=\int_{0}^{t} S(u) d u\right)$ respectively.

For $\sigma=0$ the risky asset behaves deterministically, and it is easy to explicitly derive the financial solution

$$
V_{0}(S, A, \tau)=\max \left\{\left(\frac{A}{T}-K\right) e^{-r \tau}+\frac{S}{r T}\left(1-e^{-r \tau}\right), 0\right\} .
$$

See Theorem 2 in [2] for a proof. 
We chose to consider zero dividend and continuous average in order to not complicate the situation with discontinuities, apart from the ones otherwise provided by the problem itself, even though especially the discrete averages has attracted some attention in the last few years, see for example [3, 4, 5]. Further, we chose to consider a fixed strike (instead of a floating) and an arithmetic average (instead of a geometric) since these are the predominant in the market according to [6]. Finally, we quite arbitrarily chose a zerostarting call option: A put option can be computed with a put call parity, see for example the corollary to Proposition 1 in [6], and general forwardand backward-starting options may be computed with methods similar to the ones presented here.

The BVP is a so-called degenerate parabolic problem with several degeneracies for which no closed form solutions are available. It is well known [7, e.g.] that such problems are not well adapted to solution with simple textbook numerical methods like standard explicit or implicit finite difference or uniform mesh finite element methods since such methods will generate spurious oscillations. Artificial viscosity may be useful in limiting the oscillations but the most successful methods combine this with nonuniform grids like in mesh adaptive finite element (or volume) methods as in [8]. For more literature see the extensive list of references in this paper.

Instead it has, to our knowledge, not been addressed previously in the literature exactly which of the various degenerations are dominating and neither exactly how bad the simple textbook methods actually behave. These are the two research questions that we set out to investigate in this article. For both questions we utilize exactly the simple text book numerical methods (see Section 2) that are known to not function optimally. That way we will be able to understand in what sense they do not perform and their performance will also reveal the degeneracies that are dominating the picture.

We focus on explicit finite difference methods since these are the methods that are generally attempted as a first try and since they present a significant computational cost advantage over implicit methods. (Note that we are 
dealing with 3 dimensional problems). It is possible to transform the problem from 3 dimensions to 2 [6] but this will likely obscure the influence of the various degeneracies so we do not consider that option here. The goal is not as much to solve the Asian option as it is to investigate the solutions presented by others.

\section{Simple textbook finite difference methods}

We consider what we shall call simple textbook finite difference methods for (1). We shall only consider the case $\sigma>0$ since we have a closed form solution for the no volatility case $\sigma=0$ in (2).

In [2] the differential operator in (1) is studied. It is found to be hypoelliptic (for smooth data), which guarantees smooth solutions. Further it has degenerated elliptic $S$ and $A$ parts. The degeneration in the $S$ variable comes from the fact that the coefficient $\frac{1}{2} \sigma^{2} S^{2}$ of $\partial^{2} V / \partial S^{2}$ takes the value 0 at the left boundary of $\Omega$, that is, for $S=0$. Such a degeneracy could be expected to generate oscillations in numerical solutions which might be avoided by adding some (non negative) amount $\nu_{S}^{2}$ of artificial viscosity, replacing the coefficient by $\frac{1}{2} \sigma^{2}\left(S+\nu_{S}\right)^{2}$, or alternatively by $\frac{1}{2} \sigma^{2}\left(S^{2}+\nu_{S}^{2}\right)$, but this latter will not necessarily preserve the hypoellipticity of the differential operator and hence should not be chosen. Adding artificial viscosity generally smoothens out a numerical solution, but also decreases the precision since we are now considering a different problem. The art is to add exactly the right amount to avoid the oscillations but to not deteriorate the solution too much. The degeneration in the $A$ variable is global since there is no $\partial^{2} V / \partial A^{2}$ term at all in the differential equation. The solution is similar, adding a non negative artificial viscosity $\nu_{A}^{2}$ ending up with a term $\nu_{A}^{2} \partial^{2} V / \partial A^{2}$. An artificial viscosity numerical method for (1) will then take its origin in the differential 
equation

$$
\frac{\partial V}{\partial \tau}=\frac{\sigma^{2}}{2}\left(S+\nu_{S}\right)^{2} \frac{\partial^{2} V}{\partial S^{2}}+r S \frac{\partial V}{\partial S}+\nu_{A}^{2} \frac{\partial^{2} V}{\partial A^{2}}+S \frac{\partial V}{\partial A}-r V
$$

In the most general case, $\nu_{S}$ and $\nu_{A}$ depend on $S, A$ and $\tau$, while selecting $\nu_{S}=\nu_{A}=0$ we return to the standard case without artificial viscosity. Our simple textbook finite difference methods for (1) then consist in the replacement of the various differential operators in (3) by finite difference operators on a uniform mesh with step lengths $h_{S}, h_{A}$ and $h_{\tau}$ in the $S, A$ and $\tau$ directions respectively and the consideration of the resulting equation and also the boundary conditions in (1) in the nodal points of the mesh. To qualify as a simple method, we shall allow $\nu_{S}$ to depend on $h_{S}$ and $\nu_{A}$ to depend on $h_{A}$. Instead they must be independent of $S, A$ and $\tau$. Our general finite difference method then takes the form

Find $U_{i j}^{k}$ for $i=0, \ldots, N_{S}, j=n_{A}, \ldots, N_{A}, k=0, \ldots, N_{\tau}$ :

$$
\begin{aligned}
\left(U_{\tau}\right)_{i j}^{k}= & \frac{\sigma^{2}}{2}\left(i h_{S}+\nu_{S}\right)^{2}\left(U_{S S}\right)_{i j}^{k}+r i h_{S}\left(U_{S}\right)_{i j}^{k}+\nu_{A}^{2}\left(U_{A A}\right)_{i j}^{k} \\
& +i h_{S}\left(U_{A}\right)_{i j}^{k}-r U_{i j}^{k}
\end{aligned}
$$

for $i=1, \ldots, N_{S}-M_{S}, j=n_{A}+m_{A}, \ldots, N_{A}-M_{A}, k=0, \ldots, N_{\tau}-1$, $\left.\partial \Omega\right|_{\tau=0}: U_{i j}^{0}=\max \left\{\frac{j h_{A}}{T}-K, 0\right\}$, for $i=0, \ldots, N_{S}, j=n_{A}, \ldots, N_{A}$, $\left.\partial \Omega\right|_{A=A_{\max }}: U_{i j}^{k}=\left(\frac{j h_{A}}{T}-K\right) e^{-r k h_{\tau}}+\frac{i h_{S}}{r T}\left(1-e^{-r k h_{\tau}}\right)$, for $i=0, \ldots, N_{S}, j=N_{A}-M_{A}+1, \ldots, N_{A}, k=1, \ldots, N_{\tau}$, $\left.\partial \Omega\right|_{S=0}: U_{0 j}^{k}=e^{-r j h_{\tau}} \max \left\{\frac{j h_{A}}{T}-K, 0\right\}$, for $j=n_{A}, \ldots, N_{A}-M_{A}, k=1, \ldots, N_{\tau}$,

$\left.\partial \Omega\right|_{S=S_{\max }}: U_{i, j}^{k}=\max \left\{\left(\frac{j h_{A}}{T}-K\right) e^{-r k h_{\tau}}+\frac{i h_{S}}{r T}\left(1-e^{-r k h_{\tau}}\right), 0\right\}$, for $i=N_{S}-M_{S}+1, \ldots, N_{S}, j=n_{A}, \ldots, N_{A}-M_{A}, k=1, \ldots, N_{\tau}$. 
We are using $U_{i j}^{k}$ as our approximation to $V\left(i h_{S}, j h_{A}, k h_{\tau}\right)$. Also $U_{\tau}, U_{S}$, $U_{S S}, U_{A}$ and $U_{A A}$ are finite difference approximations to the corresponding derivatives, to be described below. $M_{S}$ and $M_{A}$ are the number of boundary conditions used at (and below) $S=N_{S} h_{S}=S_{\max }$ and $A=N_{A} h_{A}=A_{\max }$ respectively. Finally $N_{\tau} h_{\tau}=T$. Note that the $A$ indexing variable $j$ is allowed to descend down to a non positive number $n_{A}$ to allow an extension of $\Omega$ in the negative $A$ direction down to $A_{\min }=n_{A} h_{A} \leq 0$. This will be utilized below.

For finite difference operators we shall consider 16 classical forward, backward and central types of consistency order 1,2 and 4 for both the first and second derivatives. (The first order central operators do not exist and are omitted). This selection makes (4) consistent with (1) provided only that $\nu_{S} \rightarrow 0$ as $h_{S} \rightarrow 0$ and $\nu_{A} \rightarrow 0$ as $h_{A} \rightarrow 0$. The difference operators are derived by simple Taylor expansion and we give here only one illustrative example:

$$
\begin{aligned}
& \delta_{F 4}^{2} f(x)=\frac{1}{12 h^{2}}\{45 f(x)-154 f(x+h)+214 f(x+2 h) \\
& -156 f(x+3 h)+61 f(x+4 h)-10 f(x+5 h)\}=f^{\prime \prime}(x)+\mathcal{O}_{h \rightarrow 0}\left(h^{4}\right) .
\end{aligned}
$$

We use the notation $\delta_{F i}^{j}$, for $i=1,2,4$ and $j=1,2$ for a forward difference operator of order $i$ for the $j$ th derivative. $\delta_{B i}^{j}$ and $\delta_{C i}^{j}$ are given along the same lines, only backward and central respectively. When considering partial derivatives, we shall indicate the derivatives with a second lower index consisting of the variable names as for example in $U_{S S}=\delta_{F 1, S S}^{2} U$. Note that there are no mixed derivatives in our problem so that one dimensional difference operators are all that is needed. We shall use a notation illustrated by the following example to clarify what difference operators have been used for what derivatives: The numerical method $F 1 \tau-B 1 C 2 S-F 1 B 4 A$ is the one where we made the following choices in (4): $U_{\tau}=\delta_{F 1, \tau}^{1} U, U_{S}=\delta_{B 1, S}^{1} U$, $U_{S S}=\delta_{C 2, S S}^{2} U, U_{A}=\delta_{F 1, A}^{1} U$ and $U_{A A}=\delta_{B 4, A A}^{2} U$. The resulting difference 
equations are hence

$$
\begin{aligned}
\delta_{F 1, \tau}^{1} U_{i j}^{k}= & \frac{\sigma^{2}}{2}\left(i h_{S}+\nu_{S}\right)^{2} \delta_{C 2, S S}^{2} U_{i j}^{k}+r i h_{S} \delta_{B 1, S}^{1} U_{i j}^{k} \\
& +\nu_{A}^{2} \delta_{B 4, A A}^{2} U_{i j}^{k}+i h_{S} \delta_{F 1, A}^{1} U_{i j}^{k}-r U_{i j}^{k} .
\end{aligned}
$$

The naming system should be clear from this example.

The available boundary conditions prohibit quite a few of the difference operator replacements. Take for example the left side of the boundary, $S=0$. Here we have access to exactly one boundary condition. Hence using $\delta_{B 1}^{2}$, $\delta_{B 2}^{1}, \delta_{B 2}^{2}, \delta_{B 4}^{1}, \delta_{B 4}^{2}, \delta_{C 4}^{1}$ or $\delta_{C 4}^{2}$ in the $S$ direction, all having stencils including the points $x, x-h_{S}$ and $h-2 h_{S}$, would make it impossible to recover solution values in all nodal points of the mesh. To recover a value at $S=h_{S}$ we need for example information about the solution in $S=-h_{S}$ (among other) and we do not have this information. Going through all boundaries it is clear that only the following difference operators are feasible:

- in the $\tau$ direction, $\delta_{F 1}^{1}$ and $\delta_{B 1}^{1}$;

- in the $S$ direction, $\delta_{F 1}^{1}, \delta_{F 2}^{1}, \delta_{F 4}^{1}, \delta_{B 1}^{1}, \delta_{C 2}^{1}, \delta_{F 1}^{2}, \delta_{F 2}^{2}, \delta_{F 4}^{2}$ and $\delta_{C 2}^{2}$;

- in the $A$ direction, $\delta_{F 1}^{1}, \delta_{F 2}^{1}, \delta_{F 4}^{1}, \delta_{F 1}^{2}, \delta_{F 2}^{2}$ and $\delta_{F 4}^{2}$.

The difference operators $\delta_{B 1}^{1}, \delta_{C 2}^{1}$ and $\delta_{C 2}^{2}$ in the $A$ direction require one boundary condition in $A=0$ which we do not have. We shall in any case try out some of these cases, extending the BVP down to $A_{\min }<0$ and imposing an artificial boundary condition in $A=A_{\min }$. We experimented with 3 artificial boundary conditions, one being $\left.V\right|_{A=A_{\min }}=0$, another being $\left.V\right|_{A=A_{\min }}=$ $\left.V_{0}\right|_{A=A_{\min }}$ (with $V_{0}$ given by (2)) and the last one being $\left.V\right|_{A=A_{\min }}=$ "whatever comes out of the difference equations replacing the difference operators being used in the $A$ direction when $A=A_{\text {min }}$ with $\delta_{F 2}^{1}$ and $\delta_{F 2}^{2}$ but leaving the difference operators in the $\tau$ and $S$ directions unaltered". (For $A \neq A_{\min }$ the correct difference operators are being used). We shall denote the 3 options 
$X \tau-Y Z S-U V A_{0}, X \tau-Y Z S-U V A_{\mathrm{f}}$ (f for formula) and $X \tau-Y Z S-U V A_{\mathrm{p}}$ (p for pde) respectively, where $X, Y Z$ and $U V$ stands for whatever operators are being used for $A \neq A_{\min }$.

All together there are 2850 possible methods, including the artificial viscosity and the 3 different artificial boundary conditions in the $A$ direction. Obviously we shall not try them all out but we shall make a careful selection of the few reasonable methods. In order to reduce the computational cost we shall restrict to explicit time stepping methods of the Type $F 1 \tau$ for the $\tau$ direction. For the $S$ and $A$ directions where we may have both first and second derivatives, we shall with a few exceptions only consider methods of the same order in the two derivatives. This is to avoid needless waste of computation time since the highest order difference operator will determine the computing time while the lowest order difference operator will likely determine the precision. Furthermore, we shall as far as possible use overlapping stencils for the difference operators for the first and second order derivatives to limit the computational complexity of the problem. This rules out for example the method $F 4 B 4$ where the two stencils have only one point in common $(S t(F 4)=\{x, x+h, x+2 h, x+3 h, x+4 h\}$ and $S t(B 4)=\{x, x-h, x-2 h, x-3 h, x-4 h, x-5 h\})$. This leads to the following selection: for the $S$ direction we shall consider methods of the Types $F 1 F 1 S, F 2 F 2 S, F 4 F 4 S$ and $C 2 C 2 S$. For a more thorough investigation of the low order methods we also consider two methods where we have different orders in the first and second order derivatives, namely $F 1 C 2 S$ and $B 1 C 2 S$. For the $A$ direction we shall consider methods of the types $F 1 F 1 A$, $F 2 F 2 A, F 4 F 4 A, C 2 C 2 A_{0}, C 2 C 2 A_{\mathrm{f}}$ and $C 2 C 2 A_{\mathrm{p}}$. This reduces the total number of possible methods to a more manageable 36 while not leaving out any "reasonable" simple textbook methods. 


\section{$3 \quad$ Numerical experiments and conclusions}

For the numerical experiments we consider a one year option $(T=1)$, with an exercise price $K=150$, time independent squared volatility $\sigma^{2}=1 \%$ and risk free market interest rate $r=5 \%$. We take $S_{\max }=1.25\left(300+M_{S} h_{S}\right) \simeq 375$ where $S \in(0,300)$ is the interval where we want to recover the solution, $300+M_{S} h_{S}$ makes room for the boundary conditions to stay above $S=300$ and the factor 1.25 makes some extra room considering that the boundary condition in $S=S_{\max }$ is actually only valid for $S_{\max } \rightarrow \infty$. $A_{\max }$ is given by $A_{\max }=\frac{4}{3}\left(K T+M_{A} h_{A}\right) \simeq 200$ where again $K T+M_{A} h_{A}$ makes room for the boundary conditions to stay above $K T$ which is where they are known. The factor $\frac{4}{3}$ gives an overlap between the $A$ interval and the part $A \geq K T$ where the exact solution to the problem is known. This is done in order to have a simple way of "checking" numerical solutions.

For checking the error we know from finance theory, that $V$ is a non negative, non decreasing function of $S$ and $A$. Hence, the greatest amplitude glb|error| of any drop in the numerical solution (for example in connection to oscillations) is a lower bound for the error $E_{\max }=\max _{i j}\left|E_{i j}^{N_{\tau}}\right|$ at the end of the computations, at reverted time $\tau=1$.

After some testing, it turns out that selecting $h_{S}=h_{A}=10$ and $h_{\tau}=0.01$ gives results that seem to be not very much influenced by rounding errors. The main results are presented in Tables 1 and 2, in all cases with zero artificial viscosity to first reveal the problems before attempting to eliminate them. We show glb|error| for each of the 36 methods together with symbolic plots indicating, in black, the approximate areas where the most dominating part of the error is located at $\tau=1$. All cases in Table 1 show

- oscillations of Type 1, centered around a vertical line $S=$ constant

with increasing amplitude as $S$ is increasing. Also all plots in Table 2 not using the boundary condition in $S=0$ show oscillations of Type 1 , 
TABLE 1: Results for the 18 cases with an artificial boundary condition in $A=A_{\min }$ and with $h_{S}=h_{A}=10, h_{\tau}=0.01$

\begin{tabular}{ccccc}
\hline \multicolumn{3}{c}{ FDM method } & $\begin{array}{c}\text { glb/error| } \\
\leq E_{\max }\end{array}$ & $\begin{array}{c}\text { symbolic } \\
\text { graph }\end{array}$ \\
\hline$F 1 \tau$ & $F 1 F 1 S$ & $C 2 C 2 A_{\mathrm{p} / 0 / \mathrm{f}}$ & $8 \cdot 10^{6}$ & $A \square \square S$ \\
$F 1 \tau$ & $F 2 F 2 S$ & $C 2 C 2 A_{\mathrm{p} / 0 / \mathrm{f}}$ & $6 \cdot 10^{12}$ & $A \square \square S$ \\
$F 1 \tau$ & $F 4 F 4 S$ & $C 2 C 2 A_{\mathrm{p}}$ & $2 \cdot 10^{18}$ & $A \square \square S$ \\
$F 1 \tau$ & $F 1 C 2 S$ & $C 2 C 2 A_{\mathrm{p} / \mathrm{o} / \mathrm{f}}$ & $300 / 200 / 120$ & $A \square \square S$ \\
$F 1 \tau$ & $B 1 C 2 S$ & $C 2 C 2 A_{\mathrm{p} / 0 / \mathrm{f}}$ & $300 / 200 / 120$ & $A \square \square S$ \\
$F 1 \tau$ & $C 2 C 2 S$ & $C 2 C 2 A_{\mathrm{p} / \mathrm{o} / \mathrm{f}}$ & $300 / 200 / 120$ & $A \square \square S$ \\
\hline
\end{tabular}

but with amplitudes decreasing as $S$ is increasing. Two cases in Table 2 $(F 1 \tau-F 1 F 1 S-F 2 F 2 A$ and $F 1 \tau-F 1 F 1 S-F 4 F 4 A)$ show, apart from the oscillations of Type 1 , also

- oscillations of Type 2, extending around the tilted curve $A \simeq 150-S$ where $V_{0}$ (see (2)) has a discontinuity in the first derivative, or rather in an area starting at the line and going down towards $(0,0)$.

For $F 1 \tau-F 1 F 1 S-F 2 F 2 A$, the oscillations of Type 1 dominate and we indicated the Type 2 oscillations in white surrounded by black lines. Later results indicate that all oscillatory solutions have oscillations of Type 2 as well as of Type 1 . The oscillations of Type 2 become evident only when the amplitude of the type 1 oscillations is sufficiently small. For three cases $(F 1 \tau-F 1 C 2 S-F 1 F 1 A, F 1 \tau-B 1 C 2 S-F 1 F 1 A$ and $F 1 \tau-C 2 C 2 S-$ $F 1 F 1 A$ ) there are no oscillations, but a small area where $E_{-}=V_{0}-U$ is significantly smaller than zero $\left(\max _{i j k}\left|\left(E_{-}\right)_{i j}^{k}\right| \simeq 12\right)$. This area is indicated by a single line. Note that $E_{-}<0$ does not indicate an error but only a 
TABLE 2: Results for the 18 cases without an artificial boundary condition in $A=A_{\min }$ and with $h_{S}=h_{A}=10, h_{\tau}=0.01$

\begin{tabular}{|c|c|c|c|c|}
\hline \multicolumn{3}{|c|}{ FDM method } & \multirow{2}{*}{$\begin{array}{c}\text { glb|error| } \\
\leq E_{\max }\end{array}$} & \multirow{2}{*}{$\begin{array}{l}\text { symbolic } \\
\text { graph }\end{array}$} \\
\hline$\tau$ & $S$ & $A$ & & \\
\hline$F 1 \tau$ & $F 1 F 1 S$ & $F 1 F 1 A$ & 600 & \\
\hline$F 1 \tau$ & $F 1 F 1 S$ & $F 2 F 2 A$ & 40 & \\
\hline$F 1 \tau$ & $F 1 F 1 S$ & $F 4 F 4 A$ & 40 & \\
\hline$F 1 \tau$ & $F 2 F 2 S$ & $F 1 F 1 A$ & $8 \cdot 10^{9}$ & \\
\hline$F 1 \tau$ & $F 2 F 2 S$ & $F 2 F 2 A$ & $10^{8}$ & \\
\hline$F 1 \tau$ & $F 2 F 2 S$ & $F 4 F 4 A$ & $6 \cdot 10^{7}$ & \\
\hline$F 1 \tau$ & $F 4 F 4 S$ & $F 1 F 1 A$ & $8 \cdot 10^{16}$ & \\
\hline$F 1 \tau$ & $F 4 F 4 S$ & $F 2 F 2 A$ & $10^{16}$ & \\
\hline$F 1 \tau$ & $F 4 F 4 S$ & $F 4 F 4 A$ & $2 \cdot 10^{15}$ & \\
\hline$F 1 \tau$ & $F 1 C 2 S$ & $F 1 F 1 A$ & 0 & \\
\hline$F 1 \tau$ & $F 1 C 2 S$ & $F 2 F 2 A$ & 8 & \\
\hline$F 1 \tau$ & $F 1 C 2 S$ & $F 4 F 4 A$ & 30 & \\
\hline$F 1 \tau$ & $B 1 C 2 S$ & $F 1 F 1 A$ & 0 & \\
\hline$F 1 \tau$ & $B 1 C 2 S$ & $F 2 F 2 A$ & 8 & \\
\hline$F 1 \tau$ & $B 1 C 2 S$ & $F 4 F 4 A$ & 30 & $A_{\square}{ }_{S}$ \\
\hline$F 1 \tau$ & $C 2 C 2 S$ & $F 1 F 1 A$ & 0 & $A_{\square} S$ \\
\hline$F 1 \tau$ & $C 2 C 2 S$ & $F 2 F 2 A$ & 8 & $A_{\square} S$ \\
\hline$F 1 \tau$ & $C 2 C 2 S$ & $F 4 F 4 A$ & 30 & ${ }^{A} \square_{S}$ \\
\hline
\end{tabular}


difference between the numerical solution and the lower bound $V_{0}$ for the solution. For more details, see Figure 1 for two examples.

For all cases with positive error bounds, a closer investigation, see Table 3 for three of the "best" examples, shows that reducing the step sizes only very slowly, if at all, reduces the error bounds. It may be possible to reduce oscillations of Type 1 , but as these are reduced, oscillations of Type 2 become visible and do not decrease with decreasing step lengths. As $h_{S}=h_{A}$ is decreased for fixed time step length we run into an instability region limiting the number of useful results. $h_{\tau}=1 / 10.000$ and $h_{S}=h_{A}=1$ has been chosen as our computational limit. It requires of the order of 36 hours on a $700 \mathrm{Mhz}$ Pentium III processor with 256 Mbyte RAM using the interpreted programming language Maple (version 8). This would correspond to less than $1 / 2$ hour in a more optimal computational setting. Because of the smooth, but very slow decrease in the error bound that even seems to turn into a slight increase we must consider these methods computationally non convergent. We further make the following observations: In order to eliminate oscillations of Type 1 and reduce the size of oscillations of Type 2 it is necessary to avoid using (inaccurate) artificial boundary conditions at $A_{\min }$. Only the accurate boundary conditions at $A=A_{\max }$ should be used. Further it is essential to use methods that utilize the boundary condition in $S=0$ as well as those in $S=S_{\max }$. The boundary condition in $S=0$ is not necessary for well posedness of the continuous problem because of the $S^{2}$ factor degeneration in the differential operator but the numerical methods behave as if the degeneracy was not there and $S=0$ was an inflow boundary. In the $A$ directions only the $F 1 F 1 A$ option eliminates the Type 2 oscillations. Higher order A operators again lead to Type 2 oscillations.

To remove the oscillations of Type 1 and 2 we now add artificial viscosity. We test with $\left(\nu_{S}, \nu_{A}\right) \in\{(\nu, 0),(\nu, \nu),(0, \nu)\}$ for $\nu \in\{0,5,10,50,100\}$, $\left(\nu^{2} \in\{0,25,100,2500,10000\}\right)$. For all methods and in all cases the oscillations either increase in amplitude or stay the same. The largest increase happens when $\nu_{A}$ is increased. Instead introducing artificial viscosity in the 
$F 1 \tau-F 1 F 1 S-F 2 F 2 A$

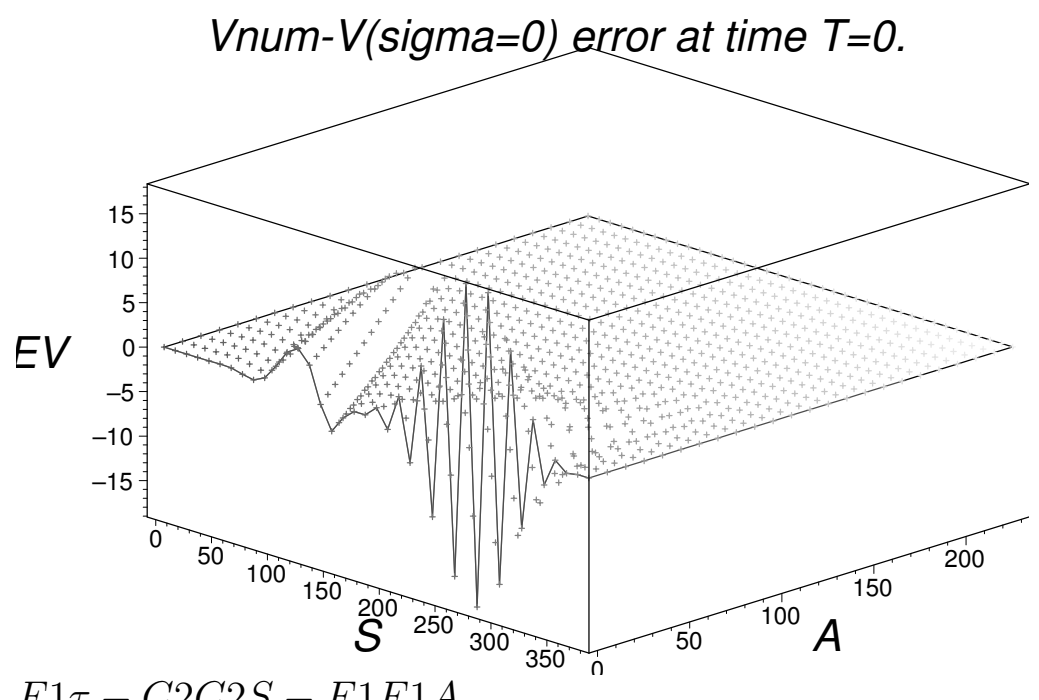

$F 1 \tau-C 2 C 2 S-F 1 F 1 A$

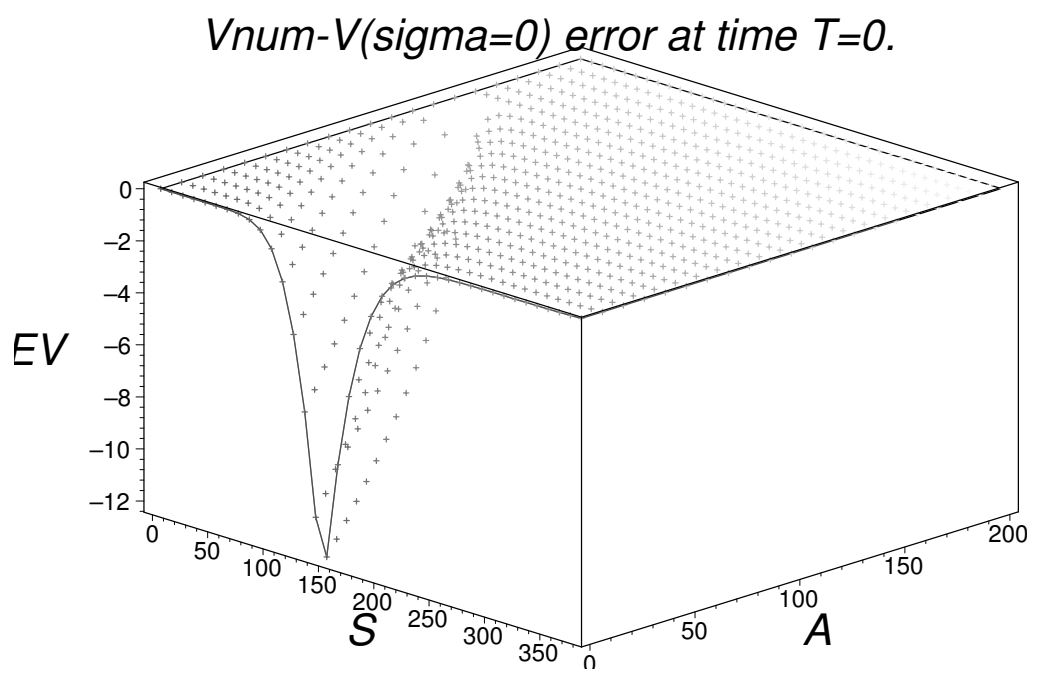

Figure 1: The difference $E V=V_{0}-U$ for two methods with and without oscillations respectively. $h_{\tau}=0.01, h_{S}=h_{A}=10$ 
TABLE 3: Results for 3 of the best performing methods for a number of time, $S$ and $A$ step lengths $\left(h_{\tau}=1 / \#\right.$ time steps, $\left.h_{S}=h_{A}\right)$. In parentheses are given glb|error| for oscillations of Type 2 whenever such are visible

glb|error| for the method $F 1 \tau-F 1 C 2 S-C 2 C 2 A_{\mathrm{f}}$

\begin{tabular}{|c|ccccccc|}
\hline$\bullet$ & 50 & 100 & 200 & 500 & 1000 & 2000 & 10000 \\
\hline 50 & 33 & 33 & 34 & 33 & 33 & 33 & 33 \\
20 & 44 & 19 & 21 & 19 & 20 & 20 & 20 \\
10 & 9000 & 120 & 20 & $10(13)$ & $8.4(14)$ & $8(14)$ & $7.2(14)$ \\
5 & $4 \cdot 10^{11}$ & $2 \cdot 10^{7}$ & 3000 & $5.6(10)$ & $2.4(11)$ & $2.8(11)$ & $3.2(12)$ \\
2 & $3 \cdot 10^{53}$ & $4 \cdot 10^{75}$ & $3 \cdot 10^{78}$ & $4 \cdot 10^{9}$ & 2400 & $11(8)$ & $11(6)$ \\
1 & $10^{84}$ & $2 \cdot 10^{139}$ & $8 \cdot 10^{217}$ & $6 \cdot 10^{305}$ & $3 \cdot 10^{242}$ & $2 \cdot 10^{8}$ & $6.4(0.5)$ \\
\hline
\end{tabular}

glb|error| for the method $F 1 \tau-F 1 F 1 S-F 1 F 1 A$

\begin{tabular}{|c|ccccccc|}
\hline$\bullet$ & 50 & 100 & 200 & 500 & 1000 & 2000 & 10000 \\
\hline 10 & 300 & 600 & 600 & 800 & 800 & 800 & 800 \\
5 & $8 \cdot 10^{16}$ & $8 \cdot 10^{16}$ & $3 \cdot 10^{17}$ & $4 \cdot 10^{17}$ & $4 \cdot 10^{17}$ & $4 \cdot 10^{17}$ & $4 \cdot 10^{17}$ \\
\hline
\end{tabular}

glb|error| for the method $F 1 \tau-F 1 F 1 S-F 2 F 2 A$

\begin{tabular}{|c|ccccccc|}
\hline$\bullet$ & 50 & 100 & 200 & 500 & 1000 & 2000 & 10000 \\
\hline 10 & $120(8)$ & $40(8)$ & $30(8)$ & $12(8)$ & $6(8)$ & $4(8)$ & $2(8)$ \\
5 & $10^{30}$ & $8 \cdot 10^{15}$ & $4 \cdot 10^{15}$ & $10^{15}$ & $8 \cdot 10^{14}$ & $3 \cdot 10^{14}$ & $7 \cdot 10^{13}$ \\
\hline
\end{tabular}

- \# timesteps $=\frac{1}{h_{\tau}}$ to the right. $h_{S}=h_{A}$ below. 
$S$ variable (increasing $\nu_{S}$ ) generally has no effect at all. Hence we reach the following conclusion: Adding constant artificial viscosities in the $S$ and/or A variables does not reduce the amplitude of oscillations and hence should not be attempted. This also shows, that it is not the degenerations in the differential operator that are creating problems. The reason for the lack of success for the artificial viscosity approach is likely that the exact solution is almost linear except for very close to the discontinuity of $V_{0}$. Hence adding second order derivative terms changes very little globally. The hope would be that there were some positive changes close to the discontinuity where the exact solution is not linear, but the experiments show that this is not the case.

We are then left with the three methods without oscillations. Here a closer investigation, to be reported elsewhere, shows that even though it is possible to reduce the error, the convergence is so slow that significant computing power and computing time is necessary to reduce the error to the order of for example one cent. The first order consistent time stepping is showing linear convergence in the $\tau$ variable. Instead both the first and second order consistent methods in the $S$ variable are showing only linear convergence in this variable. Finally, the linearly consistent method in the $A$ variable is showing a very slow $\frac{1}{2}$ order convergence in this variable.

Unless faster methods for the $A$ direction exist, the only likely solution is to use adaptive methods, like finite element methods, for the problem.

\section{References}

[1] Jens Hugger. The boundary value formulation of the Asian call option. In F. Brezzi, A. Buffa, S. Corsaro, and A. Murli, editors, Numerical mathematics and advanced applications. Enumath 2001, pages 409-418. Springer-Verlag Italia, Milano, 2003. C217 
[2] Jens Hugger. Wellposedness of the boundary value formulation of a fixed strike Asian option. Submitted to Journal of Computational Methods in Sciences and Engineering. C217, C219

[3] J. Andreasen. The pricing of discretely sampled Asian and lookback options: a change of numerical approach. Journal of Computational Finance, 2(1), 1998. C218

[4] M. A. H. Dempster. Lp valuation of exotic American options exploiting structure. Journal of Computational Finance, 2(1), 1998. C218

[5] C. Randall, E. Kant, and A. Chhabra. Using program synthesis to price derivatives. Journal of Computational Finance, 1(2), 1997/98. C218

[6] Bénédicte Alziary, Jean-Paul Décamps, and Pierre-François Koehl. A PDE approach to Asian options: analytical and numerical evidence. Journal of Banking \& Finance, 21:613-640, 1997. C218, C219

[7] R. Zvan, P. A. Forsyth, and K. R. Vetzal. Robust numerical methods for pde models of Asian options. Journal of Computational Finance, 1:39-78, 1998. C218

[8] R. Zvan, P. A. Forsyth, and K. R. Vetzal. A finite volume approach for contingent claims valuation. IMA Journal of Numerical Analysis, 21:703-731, 2001. C218 